Neueste deutschsprachige Literatur 



\section{Lucy Fricke: Töchter. Roman. Rowohlt, Reinbek bei Hamburg 2018. 240 Seiten.}

Lukas Bärfuss’ Theaterstück Alices Reise in die Schweiz (2005) stellt eines der ersten Werke der deutschsprachigen Gegenwartsliteratur dar, die das ethisch kontroverse Thema Sterbehilfe zum Gegenstand haben. ${ }^{1}$ In Anbetracht der Anzahl der seitdem erschienenen literarischen Werke, die sich damit befassen, meinte Marc Keller 2015 in der NZZ, dass man von „,einer eigentlichen Konjunktur der Sterbehilfe-Literatur“" sprechen könne. ${ }^{2}$ Auch Lucy Frickes mit dem Bayerischen Buchpreis ausgezeichneter Roman Töchter (2018) setzt sich mit Sterbehilfe auseinander und greift zudem weitere Themen auf, die in der (neuesten) Literatur von Bedeutung sind.

Töchter ist Lucy Frickes vierter Roman und bei Rowohlt erschienen. Er bildet eine Fortsetzung zu ihrem zweiten Roman Ich habe Freunde mitgebracht (2010), der von vier im Medien- und Kreativbereich tätigen Freunden handelt. Allerdings hat sich die Figurenkonstellation ,nahezu halbiert"33: Der Comiczeichner Henning macht inzwischen Trickfilme und ist vor allem aus pragmatischen Gründen mit der Radiomoderatorin Martha verheiratet (andernfalls übernähme die Krankenkasse die Kosten für deren künstliche Befruchtung nicht). Der Schauspieler Jon ist infolge der Entstellung seines Gesichts bei einem Autounfall alkoholabhängig geworden und letztlich gestorben. ${ }^{4}$ Sie sind keine handelnden Figuren mehr, sondern werden nur noch erwähnt. Der Fokus liegt auf den langjährigen Freundinnen Martha und Betty, die mittlerweile etwa vierzig Jahre alt sind. ${ }^{5}$

Betty fungiert - anders als es noch in Ich habe Freunde mitgebracht der Fall gewesen ist ${ }^{6}$ - als autodiegetische Erzählerin, das heißt als Ich-Erzählerin,

1 Zu Sterbehilfe in der Gegenwartsliteratur vgl. Caroline Welsh: Sterbehilfe und Sterbebegleitung in gegenwärtiger Literatur und Medizin. In: Zeitschrift für Germanistik 25/3 (2015), S. 499-513.

2 Marc Keller: Tod auf Verlangen. In: Neue Züricher Zeitung vom 07.02.2015, <https://www. nzz.ch/feuilleton/buecher/tod-auf-verlangen-1.18477327> vom 31.07.2020.

3 Jan Brandt: Der große deutsche Spottroman. In: die tageszeitung vom 26.05.2018, <https:// taz.de/Der-grosse-deutsche-Spottroman/!5506481/> vom 31.07.2020.

4 Anders Nina Apin, der zufolge Jon bei besagtem Autounfall ums Leben gekommen sei. Vgl. Nina Apin: Diese verdammte hartnäckige Sehnsucht. In: die tageszeitung vom 03.03.2018, $<$ https://taz.de/!5486017/> vom 31.07.2020.

5 Betty ist knapp über, Martha knapp unter vierzig Jahre alt.

6 Vgl. auch Brandt (s. Anm. 3). 
die Teil der erzählten Welt ist und auf einer ersten Ebene erzählt. Im fünften Teil des in insgesamt sieben, nicht durchnummerierten Teilen von unterschiedlicher Länge untergliederten Romans wird eine weitere intradiegetische Erzählebene eröffnet: In diesem wendet sich Betty direkt an den totgeglaubten Posaunisten Ernesto Carletti, dem einzigen der ehemaligen Freunde ihrer Mutter, den sie als Kind „wie verrückt geliebt" ${ }^{\text {"7 }}$ hat. Der siebte und finale Teil des Romans endet mit dem an seine Tochter gerichteten Abschiedsbrief von Marthas Vater Kurt, dessen Anzahl der Postskripta mit der Anzahl der Romanteile korrespondiert.

Wiebke Porombka zweifelt in ihrer Rezension des Romans in der ZEIT zu Recht an der Zuverlässigkeit der Erzählerin. ${ }^{8}$ Zum einen gehen der zu höherem Alkoholkonsum neigenden Betty im Laufe der Geschichte die Antidepressiva aus, zum anderen ist sie eine Schriftstellerin mit ausgeprägter Beobachtungsund Reflexionsgabe, der zuzutrauen wäre, dass sie die Wirklichkeit bewusst fort- und umschreibt. ${ }^{9} \mathrm{Ob}$ und zu welchem Zeitpunkt es tatsächlich zu einem solchen Umschlagen ins Unwirkliche kommt, lässt sich nicht eindeutig feststellen. Jedoch gibt es den ein oder anderen potentiellen Anhaltspunkt. So sind die ersten drei der sieben Teile von Töchter mit Zitaten aus dem Roman selbst überschrieben. Die Überschriften der daran anschließenden vier Teile, die den Zeitraum von dem Absetzen Kurts bei seiner Jugendliebe Francesca bis zu dessen Suizid auf der offenbar fiktiven griechischen Insel Lofkes ${ }^{10}$ abdecken, finden sich darin hingegen nicht wieder. Auch der fünfte Teil, in dem Betty zu Ernesto spricht und nach ihrem Versuch, sich in betrunkenem Zustand in sein vermeintliches Grab zu legen, erfährt, dass er noch am Leben ist, könnte einen solchen Einschnitt bilden. Als weiterer möglicher Wendepunkt käme der siebte Teil infrage, in dem sich Betty zunächst allein auf die griechische Insel begibt, auf die sich Ernesto auf der Flucht vor der Mafia zurückgezogen hat. Für letzteren spräche zudem, dass die Insel der einzige erfundene Handlungsort zu sein scheint und dass Betty die Reise dorthin als tief im Inneren stattfindend schildert. ${ }^{11}$

7 Lucy Fricke: Töchter. Roman. Reinbek bei Hamburg 2018, S. 19.

8 Vgl. Wiebke Porombka: ,Töchter‘. Die verschwundenen Väter. In: Die Zeit vom 22.03.2018, $<$ https://www.zeit.de/2018/13/toechter-roman-lucy-fricke-roadtrip> vom 31.07.2020.

9 Bettys Beobachtungs- und Reflexionsgabe sind auch Judith von Sternburg aufgefallen. Vgl. Judith von Sternburg: Was willst du heutzutage schreiben? In: Frankfurter Rundschau vom 13.07.2018, <https://www.fr.de/kultur/literatur/willst-heutzutage-schreiben-10962340. html> vom 31.07.2020. Vgl. auch Porombka (s. Anm. 8).

10 Der Name könnte auf das altgriechische Wort $\lambda$ عvкó $($ („weiß“) anspielen, das den Namen der tatsächlich existierenden griechischen Insel Lefkada und des realen Ortes Lefkos auf der griechischen Insel Karpathos zugrunde liegt.

11 Vgl. Fricke (s. Anm. 7), S. 161. Zieht man die Möglichkeit in Betracht, dass sich Betty die Ereignisse des siebten Teils ausgedacht haben könnte, ist es eventuell auch kein Zufall, dass Ernestos Geliebte Elena, die von der Mafia getötet worden ist, denselben Namen wie Francescas Pension - Hotel Elena - trägt, über den sich Betty und Martha zuvor gewundert haben. 
Martha und Betty sind in „,demselben trostlosen, bestenfalls kleinbürgerlichen Milieu"12 aufgewachsen. Sie haben ähnliche Probleme. Ihre Vergangenheit hat Martha dazu veranlasst, sich die Gründung einer eigenen Familie vorzunehmen, „um alles besser zu machen, um es überhaupt zu machen, glücklich werden, es durchziehen." ${ }^{\text {"13 }}$ Da sie sich ihren Kinderwunsch nicht auf natürlichem Wege erfüllen kann, unterzieht sie sich einer entsprechenden Behandlung. Obwohl diese erfolglos verläuft und Martha schon drei Abgänge hinter sich hat, gibt sie nicht auf. Während Betty keine Kinder möchte, befindet sie sich als Schriftstellerin mit Schreibblockade in einer insofern vergleichbaren Situation, als dass Schwangerschaft und Geburt geläufige Metaphern für geistige Schöpfungsprozesse darstellen. ${ }^{14}$

Betty hat in Ich habe Freunde mitgebracht als Script/Continuity gearbeitet - ein Beruf, dem auch Fricke jahrelang nachgegangen ist. ${ }^{15}$ In Töchter finanziert sie sich durch die Untervermietung ihrer Wohnung in Berlin-Kreuzberg an Touristen - an dieser Stelle übt der Roman Gesellschaftskritik und thematisiert die Gentrifizierung des Ortsteils. Zudem erfährt man, dass sie infolge von Geldnot vergeblich versucht hat, Redaktionen Texte und Konzepte zu der Finanzkrise in Griechenland anzubieten. Wie viel ihres Lebensunterhalts Betty durch das Schreiben verdient, bleibt unklar; obwohl sie bisher nur ein Buch verfasst hat und konstatiert, dass sie bald aus irgendetwas Kapital schlagen müsse, scheint es nicht nichts zu sein. Betty führt ihre Schreibblockade zum einen auf ihre Einnahme von Antidepressiva zurück und stellt dadurch einen Zusammenhang zwischen Kreativität und psychischen Erkrankungen her - eine Verknüpfung, über die auch in der Realität immer wieder spekuliert wird. Zum anderen sieht sie sie durch die gegenwärtige Lage der Welt bedingt:

Was willst du schreiben, wenn du merkst, dass du die Welt auf eine Art nicht mehr verstehst, die tief bis auf den Grund geht, wenn deine Sicherheiten wegbrechen, wenn du die Blase, in der du lebst, als Blase erkennst, die jeden Moment zerplatzen kann. [...] Was willst du schreiben, wenn alles, was du kennst, dir nur noch banal erscheint, wenn du glaubst, dass dieses Unglück nur der Anfang ist, dass etwas beginnt, von dem du keine Ahnung hast. [...] Was willst du sagen, wenn alles, was du zu wissen glaubst, das Wissen von anderen ist, die

12 Porombka (s. Anm. 8).

13 Fricke (s. Anm. 7), S. 19f.

14 Zu dieser Schwangerschafts- und Geburtsmetaphorik vgl. z. B. Christine Kanz: Maternale Moderne. Männliche Gebärphantasien zwischen Kultur und Wissenschaft (1890-1933). München 2009; Monika Schmitz-Emans: Empfängnis, Reifung, Geburt. Schwangerschaft als poetologische Metapher. In: Erfüllte Körper. Inszenierungen von Schwangerschaft. Hrsg. von Stephanie Heimgartner und Simone Sauer-Kretschmer. Paderborn 2017, S. 29-47.

15 Vgl. Brandt (s. Anm. 3). 
auch nichts mehr verstehen. [...] Was willst du da schreiben? Schreiben braucht Zeit, braucht Abstand und Verstehen und Recherche, das steht alles nicht im Vertrag, das zahlt dir auch keine Sau, die Zeit, die du brauchst, um das alles zu begreifen." ${ }^{\text {"16 }}$

In Anbetracht dieser Problematik erscheint es ihr sinnlos, saubere „Bücher, die jedem gefallen" ${ }^{\text {"17 }}$, zu verfassen, wie es diejenigen tun, die ihre schriftstellerische Tätigkeit als Arbeit auffassen und nüchtern ,,[j]eden Morgen, wenn die Kinder weg sind"“18, schreiben. Welche Art von Büchern Betty selbst verfasst beziehungsweise verfassen möchte, wird nicht dargelegt $\mathrm{t}^{19}$, aber da Martha im Laufe des Romans feststellt, dass sie wider Erwarten schwanger ist, ist zu überlegen, ob Betty ihre Schwierigkeiten in dieser Hinsicht ebenfalls überwinden und schöpferisch tätig werden kann und ob sie deshalb als Erzählerin fungiert.

Ein weiteres Problem, das Martha und Betty gemeinsam haben und das bereits im Romantitel Töchter anklingt, ist ihr Verhältnis zu ihren Eltern. In einem Interview im Deutschlandfunk sagt Fricke, dass sie die Frage nach dem Umgang mit dem Altern der eigenen Eltern dazu motiviert habe, den Roman zu schreiben. ${ }^{20}$ Sie ist der Ansicht, dass viele den Moment für ein aufrichtiges Gespräch mit ihren Eltern verpassen, und betrachtet Töchter als Möglichkeit, selbst und auch mit den eigenen Eltern zu reden. ${ }^{21}$

Einerseits beschäftigen sich Martha und Betty mit ihren Müttern, deren Entscheidungen und deren Erbe $^{22}$ :

Ich ging davon aus, dass wir die erste Generation von Frauen waren, die machen konnte, was sie wollte. Das hieß aber auch, dass wir machen mussten, was wir wollten, und das wiederum bedeutete, dass wir etwas wollen mussten. Dafür hatten unsere Mütter gekämpft. Wir sollten unsere Träume verwirklichen, wir mussten welche haben, das Scheitern wurde uns zugestanden, aber erst nachdem alles, wirklich alles versucht worden war auf dem Weg zum Glück, Psychoanalyse eingeschlossen. Nur Aufgeben war nicht vorgesehen, auch nicht, dass wir uns mit weniger zufriedengaben. Bei unseren Möglichkeiten!23

16 Fricke (s. Anm. 7), S. 58f.

17 Fricke (s. Anm. 7), S. 58.

18 Fricke (s. Anm. 7), S. 58.

19 Vgl. auch Sternburg (s. Anm. 9).

20 Vgl. Dorothea Westphal: Lucy Fricke: ,Töchter‘. „Viele verpassen den Moment, mit ihren Eltern aufrichtig zu reden“. Interview mit Lucy Fricke, Deutschlandfunk vom 17.03.2018, $<$ https://www.deutschlandfunkkultur.de/lucy-fricke-toechter-viele-verpassen-den-moment-mit-ihren.1270.de.html?dram:article_id=413308 $>$ vom 31.07.2020.

21 Vgl. Westphal (s. Anm. 20).

22 Fridtjof Küchemann ist einer der wenigen Rezensenten, der auch auf die Rolle der Mütter der Protagonistinnen eingeht. Vgl. Fridtjof Küchemann: Lucy Frickes Roman ,Töchter‘. Sturz ins Glück. In: FAZ vom 11.08.2018, <https://www.faz.net/aktuell/feuilleton/buecher/ rezensionen/belletristik/lucy-frickes-roman-toechter-15732040.html> vom 31.07.2020. 
Andererseits sind es besonders ihre Väter oder Vaterfiguren, an denen sie sich abarbeiten. Schon antike Mythen und biblische Stoffe haben Vater-TochterBeziehungen zum Gegenstand und auch in der deutschsprachigen Literatur werden sie immer wieder thematisiert; in diesem Zusammenhang ist vor allem auf Märchen und auf das bürgerliche Trauerspiel, das oftmals von VaterTochter-Verhältnissen handelt, sowie auf die literarische Rezeption von Freuds psychoanalytischem Konzept des Ödipuskomplexes zu verweisen.

Die Beziehungen von Martha und Betty zu ihren Vätern sind dadurch gekennzeichnet, dass letztere lange Zeit abwesend gewesen sind; Fricke spricht im Interview im Deutschlandfunk von der „Leerstelle, die durch Vaterlosigkeit

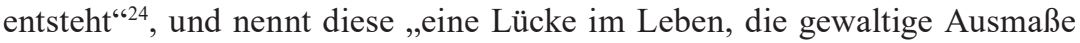
annehmen kann" ${ }^{\prime 25}$ und eine ständige Suche nach sich ziehe, auch nach Halt.

Infolge des Verlassenwerdens von Marthas Mutter, zeitweiligen Alkoholismus und einer zweiten Eheschließung hat sich Marthas Vater Kurt die ersten dreißig Jahre ihres Lebens nicht um seine Tochter gekümmert und sie beinahe vergessen. Erst das Alter, der Tod seiner zweiten Frau und seine Krebsdiagnose haben ihn dazu veranlasst, wieder regelmäßig Kontakt zu ihr aufzunehmen, und sie unterstützt ihn finanziell.

Betty verfügt über drei Vaterfiguren: ,[d]en guten, auch genannt Der Posaunist, den bösen, auch genannt Das Schwein, und den leiblichen, genannt Der Jochen. ${ }^{\text {" }}{ }^{26} \mathrm{Zu}$ ihrem leiblichen Vater, den ihre Mutter früh verlassen hat, hat sie ein höflich-distanziertes Verhältnis wie „zu einem netten Onkel“'27. Die Ehe ihrer Mutter mit dem bösen hat zwei Jahre gedauert; er scheint die vorpubertäre Betty vergewaltigt zu haben und sie sieht in ihm den Verursacher von ihren ,psychischen und sexuellen Defekten“28. Bei dem guten handelt es sich um den bereits erwähnten Ernesto. Bettys Verhältnis zu ihm ist sehr gut gewesen, bis er sie und ihre Mutter vor fünfundzwanzig Jahren verlassen hat und verschwunden ist. Über seinen vermeintlichen Tod ist sie Jahre später von ihrer Mutter informiert worden, die wiederum zufällig auf einer Feier davon gehört hat. Schließlich hat Betty seinen ältesten Freund ausfindig gemacht und von diesem erfahren, dass sich sein Grab in der italienischen Gemeinde Bellegra in der Region Latium befindet.

Zehn Jahre später - an dieser Stelle setzt die Romanhandlung in medias res ein - hält sich Betty in der Absicht, dieses Grab zu besuchen und sich von dem Totgeglaubten zu verabschieden, in Rom auf. Dort erreicht sie Marthas Anruf, der sie dazu veranlasst, unverrichteter Dinge nach Berlin zurückzukehren. Martha 
eröffnet ihr, dass Kurt selbstbestimmt sterben möchte und einen entsprechenden Termin mit einer Sterbehilfeorganisation in der Schweiz vereinbart hat, zu dem ihn seine Tochter bringen soll. Da Martha seit dem Unfall, bei dem Jons Gesicht entstellt worden ist, nicht mehr selbst Auto fährt, soll Betty das Fahren übernehmen. Sie willigt ein und die drei machen sich in Kurts altem VW Golf von seiner Heimatstadt Hannover aus über Lindau auf den Weg nach Chur. Mit der Reise in die Schweiz greift der Roman ein Motiv auf, das sich in der Sterbehilfe-Literatur oft findet, wenngleich Fricke auf eine explizite Thematisierung der verschiedenen Rechtslagen in Deutschland und der Schweiz verzichtet; Keller führt das Interesse an dem „Sterbetourismus“ auf die makabre Tatsache zurück, „dass ein Suizident sein vertrautes Umfeld hinter sich lassen muss, um sich in der

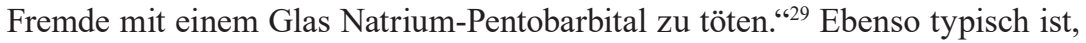
dass das Recht des Kranken auf einen selbstbestimmten Tod nicht angezweifelt wird, aber dessen Auswirkungen auf seine Angehörigen - in diesem Fall vor allem auf seine Tochter ${ }^{30}$ - ausgeleuchtet werden. ${ }^{31}$ Die Schilderung des von Kurt geplanten Todes mutet unspektakulär und durchaus realistisch an; der Roman verzichtet darauf, das Bild von einer „Schweizer Todesklinik“ ${ }^{\text {‘32 }} \mathrm{zu}$ bemühen:

„Es ist wohl eine Wohnung“, sagte sie. „Mitarbeiter sind da, ein Arzt. Kurt wird sich in ein Bett legen, wir werden uns verabschieden, dann gehe ich hinaus und warte vor der Tür. Hinter der Tür werden sie 15 Milligramm von diesem Pulver in Wasser auflösen, Natrium-Pentobarbital. Es schmeckt angeblich furchtbar. Nach wenigen Minuten fällt er in ein Koma, nach einer Viertelstunde setzt die Atmung aus. Das war's. Wir fahren zurück nach Berlin. Die Beerdigung soll nächste Woche Freitag sein, er wird verbrannt und anonym bestattet." ${ }^{\text {33 }}$

Eine Verbindung von gängigen Klischeevorstellungen von der Schweiz und Sterbehilfe, wie sie in der Sterbehilfe-Literatur häufiger anzutreffen ist ${ }^{34}$, wird lediglich insofern hergestellt, als dass Kurt kritisiert, dass die Umsetzung seines Vorhabens den Kauf einer Jahresvignette voraussetzt, und diesen Umstand mit „[t]ypisch Schweiz“35 kommentiert - an dieser Stelle wird auf das Bild von der Schweiz als Finanzplatz verwiesen, an dem auch der Tod ein Geschäft ist.

Auch nachdem Betty und Martha erfahren haben, dass Kurt den Termin nur erfunden hat, damit sie ihn zu der Pension seiner Jugendliebe Francesca in

30 Deren Mutter erfährt ebenfalls von Kurts Vorhaben, doch echauffiert sich lediglich darüber, dass er nicht einmal wie ein Mann sterben könne, und setzt assistierten Suizid mit Feigheit und Schummelei gleich. Vgl. Fricke (s. Anm. 7), S. 54.

31 Vgl. Keller (s. Anm. 2).

32 Keller (s. Anm. 2).

33 Fricke (s. Anm. 7), S. 61.

34 Vgl. Keller (s. Anm. 2).

35 Fricke (s. Anm. 7), S. 64. 
Stresa an den Lago Maggiore bringen, die sich dazu bereit erklärt hat, ihn zu pflegen, bleibt Sterbehilfe ein zentrales Thema des Romans. Zum einen wirkt das Hotel Elena wie „eine Art Hobby-Hospiz“"36 voller Medikamentenschränke und Francesca ist zwar eine ausgebildete Krankenschwester, aber scheint im Verdacht zu stehen, eine Art Todesengel zu sein. Als ihr ein Freund von der Polizei mitteilt, dass sie in Schwierigkeiten geriete, wenn eine weitere Person in ihrer Pension stürbe, wirft sie Kurt hinaus und er und Martha begeben sich zu Betty auf die griechische Insel. Dort nimmt sich Kurt schließlich mit Tabletten das Leben und hinterlässt seiner Tochter einen Abschiedsbrief. Zum anderen möchte auch Ernesto seinem Leben ein Ende setzen. Nachdem er jahrelang vergeblich auf die Rückkehr des Abgesandten der Mafia, der seine Geliebte Elena - die Ehefrau eines Mafiabosses - getötet hat, gewartet hat, versucht er Betty dazu zu überreden, ihn mit der Pistole, die dieser zurückgelassen hat, zu erschießen, da er nicht dazu in der Lage ist, es selbst zu tun. Betty weigert sich. Als sie sich ohne ihn auf den Rückweg zu ihrer Pension macht, ist sie sich nicht sicher, ob sie Schüsse hört, und Ernestos Schicksal bleibt unklar; der Titel des Romanteils - „Abschied von den Vätern“ - impliziert allerdings, dass Betty und Ernesto sich ebenfalls nicht wiedersehen werden. Beide Male kommt es in jedem Fall nicht zum assistierten Suizid.

Keller fasst die Entscheidung Angehöriger, eine nahestehende Person zum Sterben zu begleiten, als ,eine[n] Wendepunkt ihrer Entwicklung ${ }^{637}$ auf und tatsächlich stellt die Reise einen Einschnitt in das Leben der Protagonistinnen dar.

Frickes Roman lässt sich als ein sprachliches Roadmovie lesen ${ }^{38}$; Nina Apin, Peter Henning, Martina Läubli und Manuela Reichart sprechen in ihren Rezensionen gar von einer „Roadnovel“ ${ }^{39}$ Die Parallelen zu diesem in den 1960er Jahren in den USA aufgekommenen Filmgenre, dessen Handlung sich auf Landstraßen und Highways abspielt und das die Suche der Protagonisten nach Freiheit und Identität zum Gegenstand hat ${ }^{40}$, werden von Betty und Martha

36 Fricke (s. Anm. 7), S. 194.

37 Keller (s. Anm. 2).

38 Vgl. auch Sternburg (s. Anm. 9) und Westphal (s. Anm. 20).

39 Vgl. Apin (s. Anm. 4); Peter Henning: Zerzaust in die Sterbe-Schweiz. In: Spiegel Online vom 25.02.2018, <https://www.spiegel.de/kultur/literatur/lucy-fricke-mit-dem-romantoechter-zerzaust-unterwegs-in-die-sterbe-schweiz-a-1194837.html> vom 31.07.2020; Martina Läubli: Buchtipp: Zwei 40-Jährige mit einem Vaterproblem. In: NZZ am Sonntag vom 24.06.2018, <https://nzzas.nzz.ch/kultur/buchtipp-zwei-40-jaehrige-mit-einemvaterproblem-ld.1400809? reduced=true> vom 31.07.2020; Manuela Reichart: Lucy Fricke: ,Töchter‘. Am Rande des Nervenzusammenbruchs. In: Deutschlandfunk Kultur vom 09.03.2018, <https://www.deutschlandfunkkultur.de/lucy-fricke-toechter-am-rande-desnervenzusammenbruchs.1270.de.html?dram:article_id=412563 $>$ vom 31.07.2020.

40 Vgl. Reclams Sachlexikon des Films. Hrsg. von Thomas Koebner. Stuttgart 2011, S. $612-614$. 
selbst thematisiert, als sie sich nach dem Absetzen Kurts bei Francesca auf den Weg zu Ernestos Grab begeben:

„Was soll das eigentlich werden?“, fragte ich. „Thelma und Louise?“

„Die waren jung, sexy und unterdrückt“", sagte Martha. „Guck uns an, wir sind nicht mal unterdrückt.“

„Tschick?", probierte ich weiter.

„Das waren Jungs. Wir sind Frauen kurz vor den Wechseljahren. Ich hoffe, das willst du nicht vergleichen. ${ }^{(41}$

Die Selbstreflexivität des Romans wird in gewisser Weise noch gesteigert, wenn Betty Roadtrips in Filmen und Romanen als „Entwicklungsgeschichte[n] auf der Überholspur ${ }^{\text {‘42 }}$ bezeichnet, die im Gegensatz zur Langsamkeit des Lebens stehen.

Ihre Reise führt die Protagonistinnen nach Italien und nach Griechenland und somit in zwei Sehnsuchtsländer der Deutschen. Die Gemeinde Bellegra ist im 19. Jahrhundert ein Ziel von Malern der Romantik gewesen. Bettys einseitiges Gespräch mit Ernesto im fünften Teil des Romans offenbart, dass sie sich dessen bewusst zu sein scheint:

Das Dorf, das dich hervorgebracht hat, sah aus wie ein Gemälde. Du kamst aus einem scheiß Gemälde, warst der Romantik entsprungen. Warum tat sich einer wie du die deutsche Wirklichkeit an? ${ }^{43}$

Auch der deutsche Schriftsteller Wolf, der ein Haus in Bellegra besitzt, mutet wie ein Relikt aus dieser Vergangenheit an.

Gleichzeitig wird der Topos der Italien- und Griechenlandsehnsucht immer wieder relativiert und es wird auf Missstände wie den Massentourismus, das Müllproblem, den Verfall, die Naturkatastrophen, die Landflucht, die Finanzkrise, den Umgang mit Geflüchteten und die Mafia aufmerksam gemacht.

Die Folgen der ausgeprägten medialen Präsenz der beiden Länder werden ebenfalls problematisiert. So erscheint der Schiefe Turm von Pisa Betty und Martha wie „ein Postkartenbild“, „winzig“ und ,wie die Attrappe in einem Vergnügungspark “ ${ }^{44}$ Das Original büßt in Anbetracht seiner zahllosen Abbilder an Bedeutung ein - eine Problematik, die an Walter Benjamins Aufsatz Das Kunstwerk im Zeitalter seiner technischen Reproduzierbarkeit (1935) erinnert. Diese mediale Verfügbarkeit der Welt wird nicht bloß als entwertend, sondern

41 Fricke (s. Anm. 7), S. 87.

42 Fricke (s. Anm. 7), S. 107.

43 Fricke (s. Anm. 7), S. 123.

44 Fricke (s. Anm. 7), S. 106. 
sogar als lebensbedrohlich eingestuft, da sie Menschen davon abhält, sich auch einmal außerhalb ihrer Komfortzone zu begeben: „Alles konnte man sich auch am Bildschirm ansehen, in der großen Sicherheit, die tödlich war.“45

Eine besondere Rolle kommt der griechischen Insel Lofkes zu. Nachdem Martha von Ernestos Schwester in Bellegra erfahren hat, dass Ernesto sich dort vor der Mafia versteckt hat, begibt sich Betty zunächst allein dorthin; Martha kehrt zu Kurt und Francesca zurück. Betty erhofft sich von ihrem Aufenthalt auf der Insel und ihrer Auseinandersetzung mit ihrer Vergangenheit, ihr Trauma austreiben zu können. Ihre Ankunft auf Lofkes nach der Überfahrt mit einer fast leeren Fähre wird in die Nähe der Ankunft im Jenseits gerückt:

Wir legten an, die Klappe der Fähre öffnete sich, über die Lautsprecher ertönte Flötenmusik, und ich stand im Bug, musste an das Himmelstor denken, an das ich nicht glaubte. Das Versprechen eines Anfangs tat sich vor mir auf. Nichts als eine Insel war es, doch ich betrat sie mit einem so großen Schritt, als gelte es, einen Abgrund zu überspringen. Anders gesagt: Ich stolperte als Pathosbündel vom Schiff. ${ }^{46}$

Während die Fähre und die Insel Anklänge an den Fährmann Charon und an das Elysion beziehungsweise Elysium aus der antiken Mythologie darstellen, entstammt das Himmelstor der christlichen Vorstellungswelt. Die wiederholte Bezeichnung Lofkes' als Paradies oder als paradiesisch verstärkt die Assoziation mit einem Jenseitsraum. Obwohl diese Stilisierung bald aufgegeben wird, bleibt die Verknüpfung der Insel mit dem Tod bestehen: Einerseits gelangen Martha und Betty im Rahmen ihrer Reflexionen darüber, wie sie sich ihre letzten Nächte vorstellen könnten, zu dem Schluss, dass eine griechische Insel ein guter Ort zum Sterben wäre, und dieser Gedanke kommt Betty auf Lofkes erneut in den Sinn. Andererseits sterben Kurt und womöglich auch Ernesto tatsächlich dort.

Die Beschäftigung mit dem Sterben, der Grenze zwischen Leben und Tod und Jenseitsräumen rückt in der Gegenwartsliteratur verstärkt in den Fokus. ${ }^{47}$ Diese Entwicklung kann in Zusammenhang mit dem breiter gefächerten Phänomen einer „Renaissance des Religiösen“ oder gar eines „religious turn" in der neuesten Literatur ${ }^{48}$ - also einem gesteigerten Auftreten religiöser

45 Fricke (s. Anm. 7), S. 227.

46 Fricke (s. Anm. 7), S. 162.

47 Vgl. z. B. Isabelle Stauffer: Jenseits im Diesseits. Paradies und Hölle in Thomas Lehrs ,Frühling' (2001) und Sibylle Lewitscharoffs ,Consummatus' (2006). In: Zeitschrift für Germanistik 25/3 (2015), S. 551-564, hier S. 551.

48 Vgl. Albrecht Grözinger u. a.: Religion und Gegenwartsliteratur. Spielarten einer Liaison. Einleitendes zum Band und zur Thematik. In: Religion und Gegenwartsliteratur. Spielarten einer Liaison. Hrsg. von denselben. Würzburg 2009, S. 1-19, hier S. 2; Silke Horstkotte: 
Themen und Motive in der Gegenwartsliteratur - gesehen werden. In Töchter lässt sich dieses Phänomen ebenfalls beobachten. ${ }^{49}$ Betty sagt von sich selbst, dass sie nicht an Gott und den Himmel glaube. Dennoch greift sie bei ihren Schilderungen und bei ihrer Selbstinszenierung, die nicht frei von Ironie sind, immer wieder auf Bilder aus dem religiösen Bereich zurück: So veranlasst sie der Anblick einer mindestens sechs Meter hohen, bronzenen Tür im Pantheon in Rom zu dem Ausspruch „Wenn der Himmel solche Türen hatte, käme ich da nie rein ${ }^{\text {" } 50}$ und in Anbetracht ihrer Vergangenheiten, über die sie nie gesprochen haben, kommt es ihr so vor, als wären sie und Martha ,vom Himmel gefallen, hinabgestoßen eher." ${ }^{\text {"51 }}$ Dadurch scheint sie eine Verbindung zu gefallenen Engeln herzustellen, denen eine Rückkehr in den Himmel verwehrt ist. Wiederholt wird auf die helfende und rettende Wirkung Gottes und des Glaubens an ihn verwiesen - eine Option, mit der Betty ihre Schwierigkeiten hat. Ernesto ist nach Elenas Tod in einem Kloster aufgenommen worden und seitdem als „Hausmeister Gottes" ${ }^{\text {“52 }}$ tätig. Besonders deutlich treten die religiösen Bezüge bei der Ankunft Kurts und Marthas auf Lofkes hervor. Diese fällt mit dem Beginn des orthodoxen Osterfestes zusammen und die beiden werden von Kirchenglocken, Feuerwerk und „Christós Anésti“-Rufen begrüßt. Der auferstandene Jesus Christus bildet einen extremen Gegensatz zu dem todkranken Kurt. Es ist zu überlegen, wie die Tatsache, dass Kurt und möglicherweise auch Ernesto ausgerechnet an Ostern Suizid begehen, zu interpretieren ist. Während sie in Kurts Fall - vor allem in Anbetracht des ihm für das kommende Jahr vorhergesagten Glücks im Rahmen einer griechischen Ostertradition und des Tenors seines Abschiedsbriefs - nahelegen könnte, dass es ein Leben nach dem Tod geben könnte, scheint sie bei Ernesto eher eine Kontrastfunktion zu haben. Die Begegnung mit diesem in gewisser Weise ebenfalls von den Toten Auferstandenen erweist sich als desillusionierend und seine Resurrektion ist nicht von Dauer.

Zusammenfassend lässt sich festhalten, dass Frickes Roman zu der gegenwärtigen Tendenz der Sterbehilfe-Literatur zu rechnen ist und sich ausgehend von der Thematik des assistierten Suizids mit Aspekten des Sterbens im Allgemeinen und somit mit einem weiteren wichtigen Gegenstand der Gegenwartsliteratur auseinandersetzt. Zugleich nimmt er Bezug auf traditionelle

Heilige Wirklichkeit! Religiöse Dimension einer neuen Fantastik. In: Poetiken der Gegenwart. Deutschsprachige Romane nach 2000. Hrsg. von derselben u. a. Berlin u. a. 2013, S. 67-82, hier S. 82; Georg Langenhorst: „Ich gönne mir das Wort Gott“. Gott und Religion in der Literatur des 21. Jahrhunderts. Freiburg u. a., S. 9-17.

49 Dass sich in dem Roman zahlreiche religiöse Metaphern und Motive finden, stellt auch Brandt fest. Vgl. Brandt (s. Anm. 3).

50 Fricke (s. Anm. 7), S. 10.

51 Fricke (s. Anm. 7), S. 29.

52 Fricke (s. Anm. 7), S. 209. 
Topoi wie das Vater-Tochter-Verhältnis oder die Italien- und Griechenlandsehnsucht und modernisiert sie. Darüber hinaus geht er auf aktuelle Themen wie die Kinderwunschbehandlung oder das Schreiben in einer Zeit, die von Unübersichtlichkeit und Unsicherheit geprägt zu sein scheint, ein.

Veronika Born Katholische Universität Eichstätt-Ingolstadt 
